and establish a correlation to the severity of risk factors or clinical condition of the neonate.

Method We prospectively collected data of neonates at risk over a 2 month period. Perinatal risk factors were derived from the National Institute of Clinical Excellence (NICE) guideline. Identified neonates had blood cultures, FBC and CRP collected prior starting antibiotics. CRP was repeated 24 hrs later. Lumbar puncture was performed in neonates with CRP $\geq 30$.

Results A total of 73 babies were identified, out of which 9 $(12.3 \%)$ were late preterm. 19 out of $73(23 \%)$ neonates had elevated CRP and were treated for 5 to 7 days. 10 out of these $23(43.4 \%)$ neonates had maternal pyrexia as a risk factor. All babies have been clinically well and managed on postnatal ward. Blood cultures in all identified neonates have been negative. 30 out of $54(55 \%)$ neonates with normal CRP stayed in hospital for more than 48 hrs awaiting blood culture results and clinical reviews.

Conclusion The identification of neonates at risk is a combination of perinatal risk factors, which are neither specific nor sensitive and infant laboratory values. This approach leads to clinicians treating well appearing infants for an extended period of time.

\section{PO-0532 ACTIVE MANUKA HONEY DRESSINGS IN BABIES REQUIRING ADMISSION TO NICU; ASSESSING THE FEASIBILITY OF A RANDOMISED TRIAL}

${ }^{1} \mathrm{~J}$ Dorling, ${ }^{1} \mathrm{~V}$ Lee, ${ }^{2} \mathrm{~A}$ Leslie, ${ }^{2} \mathrm{D}$ McInnes. ${ }^{1}$ Child Health Obstetrics and Gynaecology, University of Nottingham, Nottingham, UK; ${ }^{2}$ Neonatal Service, Nottingham University Hospitals, Nottingham, UK

\subsection{6/archdischild-2014-307384.1176}

Honey dressings have important anti-infective and wound healing properties in adults but have not been adequately studied in newborns. Osmotic and antinflammatory effects are believed to be important mechanisms of action. We carried out a pre-trial feasibility study and assessed safety and acceptability to staff and parents.

Methods Following informed parental consent, babies were recruited to an observational study of Active Manuka Honey Dressings (Advancis Activon Tulle). Parents completed a questionnaire examining the acceptability and performance of the dressings and staff members who used the dressings filled out a similar questionnaire. The study was fully approved by the Nottingham 2 Research Ethics Committee and funded by Bliss.

Results 28 wounds (8 different types) were dressed using the Active Manuka Honey dressings. Median gestational age at birth was 25 weeks (range 166-284 days), median age at entry into the study was 6 days (2-64 days). Median birth weight, $770 \mathrm{~g}$, $(500-5305 \mathrm{~g})$. Other parents declined to take part due to not wishing to disturb the dressing applied whilst considering taking part. Surgeons expressed strong preferences for other dressings. Analysis of predictive variables for time to wound healing did not identify any associations

Conclusions Honey Dressings were easy to apply, well tolerated, with little pain on application or removal but there were fewer wounds than expected.

No infant required escalation of pain relief or developed hyperglycaemia felt to be due to the dressings. These results suggest that Honey dressings are safe but that a trial may be challenging.

\section{PO-0533 NEONATAL BLOOD CULTURE: SURVEY OF BLOOD CULTURE BOTTLE USE IN UK NEONATAL UNITS}

${ }^{1} \mathrm{~J}$ Egyepong, ${ }^{2} \mathrm{D}$ Bell, ${ }^{3} \mathrm{~J}$ Amenu. ${ }^{1}$ Neonatal Intensive Care Unit, Luton and Dunstable University Hospital, Luton, UK; ${ }^{2}$ Medical Student, University College London, London, UK; ${ }^{3}$ Paediatric Intensive Care Unit, Kings College Hospital, London, UK

\subsection{6/archdischild-2014-307384.1177}

Introduction Neonatal sepsis carries a high morbidity and mortality. Blood culture is the current 'gold standard' investigation. The results obtained is of vital diagnostic, therapeutic, surveillance and prognostic importance. Traditionally in the past, paired samples were used, however with the decrease in anaerobic infections, from the 1980s and current Health Protection Agency (UK) figures on bacteria causing neonatal sepsis, this practice is changing with calls to abandon anaerobic blood cultures.

Objectives Survey of all Neonatal units in UK to determine-

- Whether single (aerobic) or paired (aerobic and anaerobic) bottles are used.

- If single, whether there are special occasions when anaerobic cultures are included.

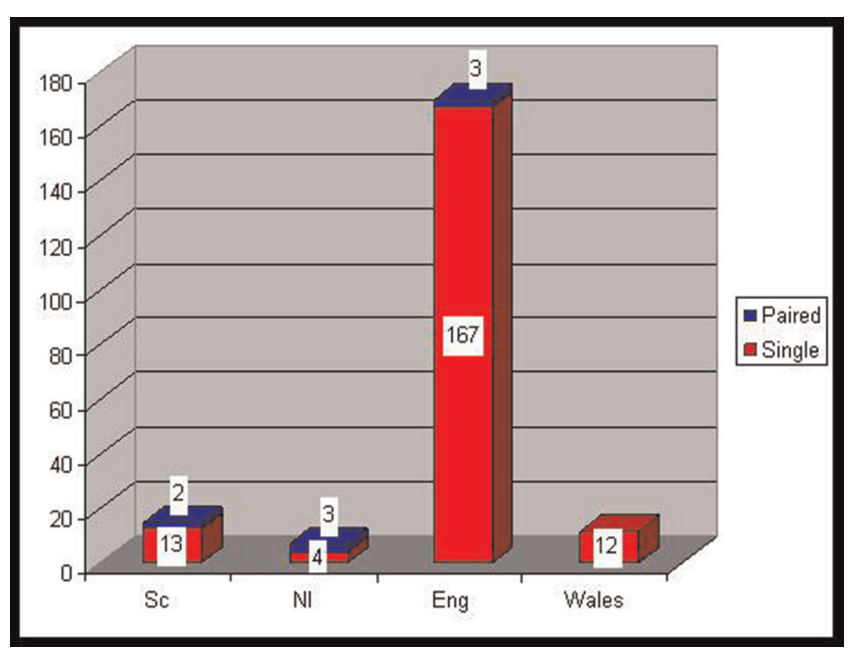

Abstract P0-0533 Figure 1

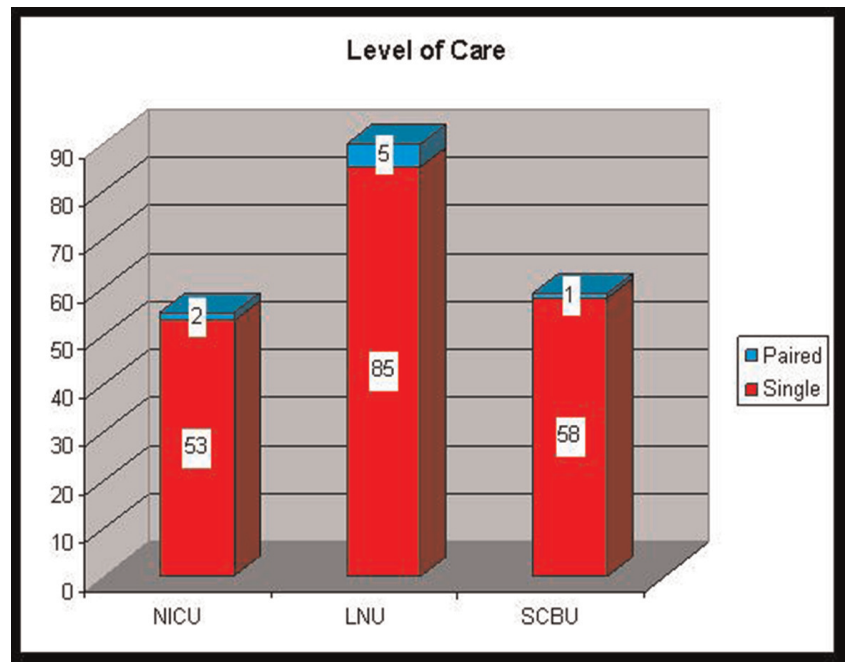

Abstract P0-0533 Figure 2 
- Any variations in practice according to the level of care and in the different nations of the UK.

\section{Methods}

- Read Literature on neonatal blood culture bottle use to inform questionnaire design.

- Telephone survey Feb-March 2014.

- Interviewee: medical staff or nurse-in-charge only.

Results

- All 204 neonatal units were surveyed.

- Number using single $=197 / 204$ (96.1\%); paired $=8 / 204$ (3.9\%).

- 11/197 using single, occationally use paired.

- Paired: England 3/170 (1.8\%); Scotland 2/15 (13.4\%); Wales $0 / 12$ and NI 3/7 (42\%) (Figure 1).

- Level of care: NICU 2/55(3.6\%), LNU 5/90(5.6\%) and SCBU 1/59(1.7\%) (Figure 2).

\section{Conclusions}

- There is general uniformity in the type of bottle used- single aerobic bottles.

- As the 'gold standard' investigation for neonatal sepsis, it needs to follow evidence-based traditions to improve yield.

- There is a need to be aware of when an anaerobic bottle should be included rather than a 'box-standard' approach.

\section{PO-0534 PREDICTIVE VALUES OF PRENATAL AND NEONATAL TESTING INDICATIONS FOR THE DIAGNOSIS OF CONGENITAL CYTOMEGALOVIRUS (CMV) INFECTION}

${ }^{1} \mathrm{~S}$ Eventov-Friedman, ${ }^{2} \mathrm{~S}$ Zaharan, ${ }^{3} \mathrm{M}$ Geal-Dor, ${ }^{4} \mathrm{D}$ Wolf, ${ }^{1} \mathrm{~B}$ Bar-Oz. ${ }^{1}$ Neonatology, Hadassah-Hebrew University Medical Center, Jerusalem, Israel; ${ }^{2}$ Faculty of Medicine, Hadassah-Hebrew University Medical Center, Jerusalem, Israel; ${ }^{3}$ Audiology, HadassahHebrew University Medical Center, Jerusalem, Israel; ${ }^{4}$ Clinical Virology, HadassahHebrew University Medical Center, Jerusalem, Israel

\subsection{6/archdischild-2014-307384.1178}

Background and aims Despite its major public health impact, there is currently no official screening policy to identify Congenital CMV Infection (CCI) and neonatal testing is based mostly on clinical indications. We aimed to characterise the currently employed clinical indications for CMV testing in neonatal urine samples, and to examine their positive predictive values.

Methods All prenatal and neonatal records of newborns that had urine sample analysed for congenital CMV between 2009 and 2013 at the Virology Laboratory, Hadassah Medical Centre, Jerusalem, Israel, were retrospectively reviewed. The clinical indications for CCI evaluation were obtained, and their positive predictive values were determined.

\begin{tabular}{llll}
$\begin{array}{l}\text { Abstract PO-0534 Table 1 } \\
\text { congenital CMV infection }\end{array}$ & \multicolumn{4}{l}{ Clinical indications tested for } \\
\hline Clinical indication & \% of all & Positive predictive \\
Maternal seroconversion & urine samples & value $(\%)$ & $95 \% \mathrm{Cl}$ \\
IUGR/SGA & 41.7 & 7 & {$[5.9-7.6]$} \\
Failed OAE screening & 38 & 1.6 & {$[0.8-2.7]$} \\
Head circumference $<$ 10th percentile & 10.4 & 2.9 & {$[1.1-6.5]$} \\
Thrombocytopenia & 9.7 & 1.9 & {$[0.5-5.4]$} \\
\hline
\end{tabular}

Results Of 1625 neonates tested, 58 (3.56\%) were positive for CCI. The leading clinical indications for testing and their positive predictive values are shown in the Table.

Interestingly, we further identified differences in the distribution and predictive values of the clinical indications between ethnic subpopulations.

Conclusions As suspected maternal CMV infection during pregnancy yielded the highest predictive value and detection rate of CCI, we suggest that in the absence of general neonatal screening policy, prenatal maternal CMV screening should be considered as a primary option for the early identification of CCI. The cost-benefit of this screening approach needs further evaluation.

\section{PO-0535 SUSTAINABLE REDUCTION OF POSITIVE BLOOD CULTURES IN A TERTIARY NEONATAL INTENSIVE CARE UNIT: IMPACT OF INFECTION PREVENTION AND CONTROL MEASURES OVER A 5 YEAR PERIOD}

${ }^{1} S$ Fang, ${ }^{2} V$ Longstaff, ${ }^{1} \mathrm{E}$ Vanderpool, ${ }^{1} \mathrm{~S}$ Husain, ${ }^{2} \mathrm{~A}$ Claxton, ${ }^{3} \mathrm{M}$ Millar. ${ }^{1}$ Neonatal Unit, Homerton University Hospital, London, UK; ${ }^{2}$ Microbiology Infection Control, Homerton University Hospital, London, UK; ${ }^{3}$ Microbiology, Barts and London School of Medicine and Dentistry Queen Mary Colleage London, London, UK

\subsection{6/archdischild-2014-307384.1179}

Background and aims Infection is one of the major causes of mortality and morbidity amongst preterm babies in neonatal intensive care units (NICUs). Preterm babies have immature immune systems, poor skin integrity and have repeated invasive procedures making them vulnerable to blood stream infections. Hand hygiene, low nurse: patient ratios, environmental colonisation (especially of water systems) and injudicious use of antibiotics, all contribute to infection in preterm babies.

The NICU at Homerton University Hospital, is a large tertiary level unit serving a high-risk population. In October 2005, we discussed the Epic Guidelines and introduced a number of infection care bundles with the aim to reduce the number of positive blood cultures.

Method From 2005 to 2007, we adapted the adult visual inspection score (VIP) for peripheral IV cannulae. From 2008 to 2010 the care bundles included dedicated cleaned trollies with sterile packs for insertion of intravenous cannulas, blood cultures and obtaining blood samples from arterial lines and we used a closed system of suctioning and closed system to sample arterial blood. We used sterile cotton wool for taking blood from heel prick. In May 2010 we introduced 0.5\% chlorhexidine for all

\section{Blood cultures positive for coagulase negative Staph}

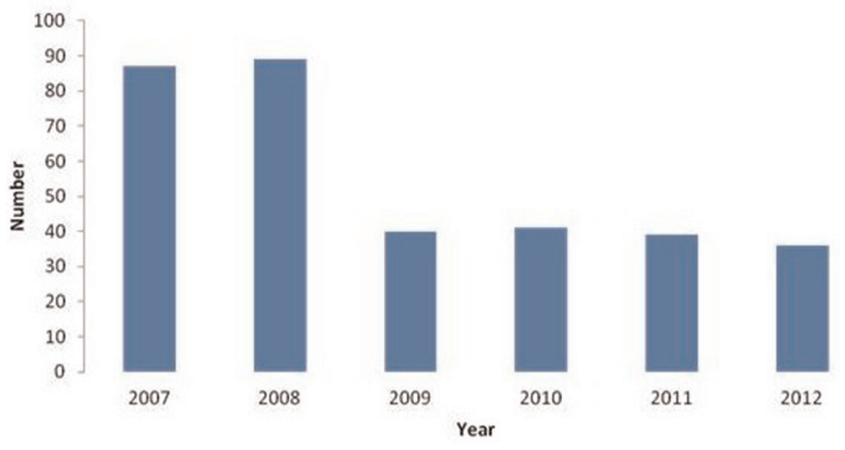

Abstract P0-0535 Figure 1 